\title{
Observations and 3D MHD simulations of a solar active region jet
}

\author{
C. Gontikakis ${ }^{1}$, V. Archontis ${ }^{2}$, and K. Tsinganos ${ }^{3}$ \\ 1 Research Center for Astronomy and Applied Mathematics, Academy of Athens, 4 Soranou Efessiou Str., Athens 11527, Greece \\ e-mail: cgontik@academyofathens.gr \\ 2 School of Mathematics and Statistics. St. Andrews University, St. Andrews, KY16 9SS, UK \\ 3 Section of Astrophysics, Astronomy and Mechanics, Department of Physics, University of Athens, Panepistimiopolis, \\ Zografos 157 84, Athens, Greece
}

Received 30 July 2009 / Accepted 22 September 2009

ABSTRACT

\begin{abstract}
Aims. We study an active region jet originating from NOAA 8531 on May 15 1999. We perform 3D MHD numerical simulations of magnetic flux emergence and its subsequent reconnection with preexisting magnetic flux. Then, we compare the physical properties of the observed jet with the reconnecting outflow produced in the numerical model.

Methods. We report observations of this jet using a series of TRACE $171 \AA$ A filtergrams, simultaneous observations from SUMER in Ne VIII 770 ̊ and C IV $1548 \AA$ as well as MDI magnetograms. In the numerical simulation, the full compressible and resistive MHD equations are solved, including viscous and Ohmic heating.

Results. A high-velocity upflow $\left(\simeq 100 \mathrm{~km} \mathrm{~s}^{-1}\right)$ is observed after the emergence of new magnetic flux at the edge of the active region. The jet is recorded over a range of temperatures between $10^{5} \mathrm{~K}$ and $1.5 \times 10^{6} \mathrm{~K}$. In our numerical experiments, we find that the jet is the result of magnetic reconnection between newly emerging flux and the preexisting magnetic field of the active region.

Conclusions. The hot and high-velocity bidirectional flows occur as a result of the interaction between oppositely directed magnetic fields. Observations and numerical results are strongly suggestive of effective reconnection process being responsible for producing jets when emerging flux appears in solar active regions.
\end{abstract}

Key words. magnetohydrodynamics (MHD) - methods: numerical - Sun: activity - Sun: corona - Sun: magnetic fields

\section{Introduction}

Jets are transient, thin, elongated features that occur mainly in polar coronal holes and at the edges of active regions. They have been observed in various wavelengths (X-ray, EUV and $\mathrm{H} \alpha$ ) by the instruments on board Yohkoh, Stereo and Hinode satellites (Shimojo et al. 1996; Chae et al. 1999; Chifor et al. 2008a; Patsourakos et al. 2008). Jets may appear recurrently (Chifor et al. 2008b) over bright points and strong flows are measured along their length. These bright points, which often are associated with microflaring activity, may correspond to emerging magnetic flux from below the photosphere (Canfield et al. 1996).

In many numerical simulations (Yokoyama \& Shibata 1996; Moreno Insertis et al. 2008; Archontis et al. 2005), the process of reconnection between an emerging sub-photospheric magnetic flux and preexisting magnetic field in the ambient atmosphere has been used to reproduce solar-like jets. These are reconnection jets where the plasma acceleration is due to the slingshot effect (Shibata 1996). Other studies (Shimojo et al. 2001; Chifor et al. 2008b) suggest that an X-ray jet is the evaporation flow produced by e.g. flaring activity in the close vicinity of a largescale loop. Observations and numerical models indicate that a fraction of jets exhibit helical structure undergoing untwisting (Canfield et al. 1996; Patsourakos et al. 2008; Pariat et al. 2009).

In this Letter we present observations of jets occurring close to an active region, cospatial with a flux emergence event. We use the observations to determine the kinetic and thermodynamic properties of the jets. Then, we briefly present the first results of a systematic effort to couple 3D MHD simulations of flux emergence and associated magnetic reconnection with observations of solar active region jets. Our numerical results show that the physical properties of the reconnecting outflows produced in the simulations are in good qualitative and quantitative agreement with the corresponding properties of jets observed in solar active regions.

\section{Observations}

The jet under study occured in the active region NOAA 8541 on May 15, 1999. The event, followed over time in TRACE $171 \AA$, $\left(0.8-1.5 \times 10^{6} \mathrm{~K}\right)$ image series, with one minute cadence, starts as a bright compact area (subflare) that forms at 13:12 UT and less than 40 min later, at 13:53 UT, a jet emerges. The jet is observed recurrently up to 14:55 UT when TRACE observations stop (Fig. 1a-g). The bright area at the jet footpoint is cospatial with a new magnetic flux emergence, near the main active region negative polarity, recorded in the MDI magnetograms at 14:24 UT (Fig. 1 panel 1). Note that this polarity is absent in the 12:48 UT magnetogram. SUMER observed the jet at 14:44 UT when performing a raster of the active region recording the chromospheric Si II $1533 \AA,\left(10^{4.4} \mathrm{~K}\right)$ the transition region doublet $\mathrm{C}$ IV $1548 \AA, 1550 \AA$, $\left(10^{5} \mathrm{~K}\right)$ (Fig. $\left.1 \mathrm{i}, \mathrm{j}\right)$ the coronal Ne VIII $770 \AA\left(10^{5.8} \mathrm{~K}\right)$ (Fig. $\left.1 \mathrm{~g}, \mathrm{~h}\right)$ and the chromospheric continuum at $1527 \AA$, (line formation temperatures from Arnaud \& Rothenflug 1985).

We applied standard treatment for the SUMER data (Teriaca et al. 1999). Moreover, for the dopplergrams derived with 


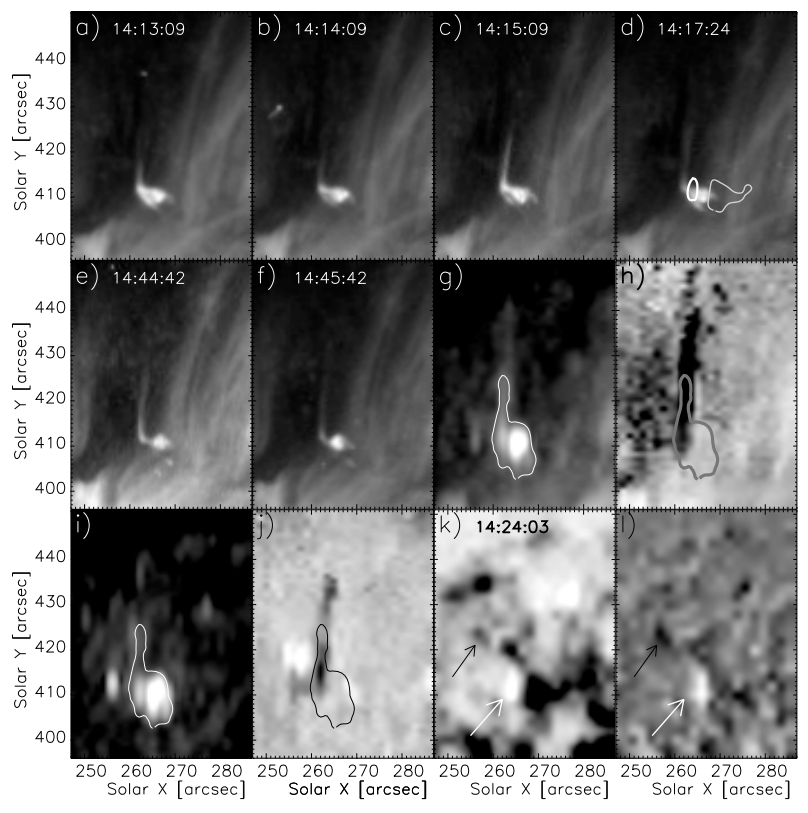

Fig. 1. Summary of the TRACE $171 \AA$ (panels a-f), SUMER (panels $\mathbf{g}-\mathbf{j}$ ) and MDI (panels $\mathbf{k}, \mathbf{l}$ ) observations of the jet. SUMER panels show: the Ne VIII $770 \AA$, intensity g), dopplergram h), C IV $1548 \AA$, intensity i) and dopplergram j). Panels k) and l) show the MDI magnetogram at 14:24 UT and the difference between the 14:24 and 12:48 magnetograms. In panel h) Doppler shifts range from $-100 \mathrm{~km} \mathrm{~s}^{-1}$ (blueshift, black) to $40 \mathrm{~km} \mathrm{~s}^{-1}$ (redshift, white) while in panel $\mathbf{j}$ ), they range from $-20 \mathrm{~km} \mathrm{~s}^{-1}$ (blueshift, black) to $33 \mathrm{~km} \mathrm{~s}^{-1}$ (redshift, white) and in panel $\mathbf{k}$ ), magnetic fields range from -450 Gauss (black) to 50 Gauss (white). The jet in panels a) to d) has its maximum brightness. In panels e) and f) the jet is observed simultaneously with the SUMER observations of panels $\mathbf{g}$ ) to $\mathbf{j}$ ). The intensity isocontours of panel $\mathbf{g}$ ), when overlaid on panel $\mathbf{h}$ ) include upward motions along the jet (black on the dopplergram) and downward motions at the bright area. In panels k), l) the two arrows indicate the emerging magnetic flux. In panel d), the white isocontour shows the emerging flux while the grey isocontour shows a pre-existing, negative polarity.

SUMER, a reference velocity is computed by using chromospheric lines with an uncertainty of $\pm 6 \mathrm{~km} \mathrm{~s}^{-1}$ (see Gontikakis et al. 2006). The proper motions along the jet, measured from the variation of the jet length in successive $171 \AA$ filtergrams from $14: 42$ UT to $14: 47 \mathrm{UT}$, are of $\simeq 90 \mathrm{~km} \mathrm{~s}^{-1}$. At the same time, the average blue shifts measured along the jet, using Ne VIII $770 \AA$, was of $-60 \pm 6 \mathrm{~km} \mathrm{~s}^{-1}$, with blueshift/redshift corresponding to negative/positive velocities through our work. In Gontikakis et al. 2006, we considered that these were two components of the same plasma flow, one in the plane of the sky (the proper motions) and one along the line of sight (the Doppler shifts). Their combination provided a full velocity of $117 \mathrm{~km} \mathrm{~s}^{-1}$ and an angle of the jet of $26^{\circ}$ to the normal on the solar surface. The jet is apparent as blueshifted with velocities -10 to $-20 \mathrm{~km} \mathrm{~s}^{-1}$ in the C IV $1548 \AA$ line. The bright structure, which appears to be composed of small unresolved loops in one 171 image (Fig. 1 panels c, d), appears redshifted in the C IV and Ne VIII lines by $15 \pm 6 \mathrm{~km} \mathrm{~s}^{-1}$ (Fig. 1 panel j). The upflow together with the lower, bright feature, form a structure with an "L-like" shape.

Applying the emission measure loci technique (Schmelz et al. 1996), the jet plasma seems to have a transition region component observed in the C IV 1548 and $1550 \AA$ lines with an emission measure of $\simeq 10^{42.5} \mathrm{~cm}^{-3}$ and a coronal component appearing in Ne VIII $770 \AA$, and in 171 filtergrams with a temperature range of $600000 \mathrm{~K}$ to $1.5 \times 10^{6} \mathrm{~K}$ with an emission measure of $10^{42 \pm 0.5} \mathrm{~cm}^{-3}$. The jet does not appear in the chromospheric temperature line of Si II $1533 \AA$, nor in the continuum, restricting the temperature range of the jet plasma only in the transition region and coronal temperatures. From the emission measure value, a jet width of $w \simeq 2^{\prime \prime}$ derived from 171 filtergrams and a unity filling factor, we can estimate the coronal plasma electron density as $n_{\mathrm{e}} \simeq 2 . \times 10^{9} \mathrm{~cm}^{-3}$ within a factor of 2 .

\section{Numerical model}

The numerical experiment was performed in a three-dimensional Cartesian box of dimensionless size using a 3D version of the Lare shock-capturing code (see Arber et al. 2001). The time-dependent, resistive and compressible MHD equations are solved by a Lagrangian remap scheme. The energy equation includes small shock viscosity and Joule dissipation terms. The resistivity is uniform, with a value of $\eta=10^{-3}$. Radiative transfer and heat conduction are not included. The equations are solved in a uniform grid of $(256,256,256)$ in the two horizontal $(x, y)$ and the vertical $(z)$ directions, respectively. The dimensionless size of the domain is $[-80,80] \times[-80,80] \times[-20,105]$ in the $(x, y, z)$ directions. The physical size of the box is $27.2 \times 27.2 \times$ $22.9 \mathrm{Mm}$. We use a uniformly spaced coordinate system in all directions. The background stratification includes a constantentropy region of $3.4 \mathrm{Mm}$ thickness that simulates the uppermost layers of the solar interior just below the surface; an isothermal layer $(T=6500 \mathrm{~K})$ with thickness $1.7 \mathrm{Mm}$ that represents the photosphere and the chromosphere and an isothermal corona at $T=10^{6} \mathrm{~K}$ with a thickness of $16.1 \mathrm{Mm}$. The photosphere and chromosphere are joined to the corona through a transition region with a steep temperature gradient. Our aim is to study the interaction of an emerging bipolar region and a small active region. The latter is formed by the dynamic rise of a flux rope in the photosphere and its lateral expansion into the higher atmosphere. The appearance of new magnetic flux at the outskirts of the preexisting active region is simulated by the emergence of another flux rope, smaller in size and weaker in field strength.

Rising buoyant magnetic flux tubes, subject to fragmentation by the Rayleigh-Taylor and Kelvin-Helmholtz instabilities, have been treated in Tsinganos (1980). In the present simulations, the flux ropes are considered as curved loops with footpoints anchored in the deeper layer of the convection zone. We use the same boundary and initial conditions as the work by Hood et al. 2009, who studied the emergence of one toroidal flux tube in a stratified atmosphere. Thus, the curved loops in our experiment are made buoyant by setting the temperature inside the loops equal to the temperature of the external medium. The excess pressure is

$p=p(r)=B_{0}^{2} \mathrm{e}^{-2 r^{2} / a^{2}}\left(\alpha^{2} a^{2}-2-2 \alpha^{2} r^{2}\right) / 4$.

Where $B_{0}$ is the axial magnetic field strength, $a$ is the minor radius of the flux tube, $r$ is the radial distance and $\alpha$ is the twist (parameter). The resulting density deficit leads to the buoyant rise of the loops to the photosphere. At the beginning of the experiment, the toroidal loops are located below the photosphere, with axes parallel to the $y$ direction. The fieldlines are uniformly twisted around the main axis of each loop. The twist is such that the average pitch of the fieldlines to the axial direction is 40 degrees. The twist is right-handed in both tubes. Thus, the fieldlines of the tubes may form rotational discontinuities and reconnect when they come into contact. One of the loops has 


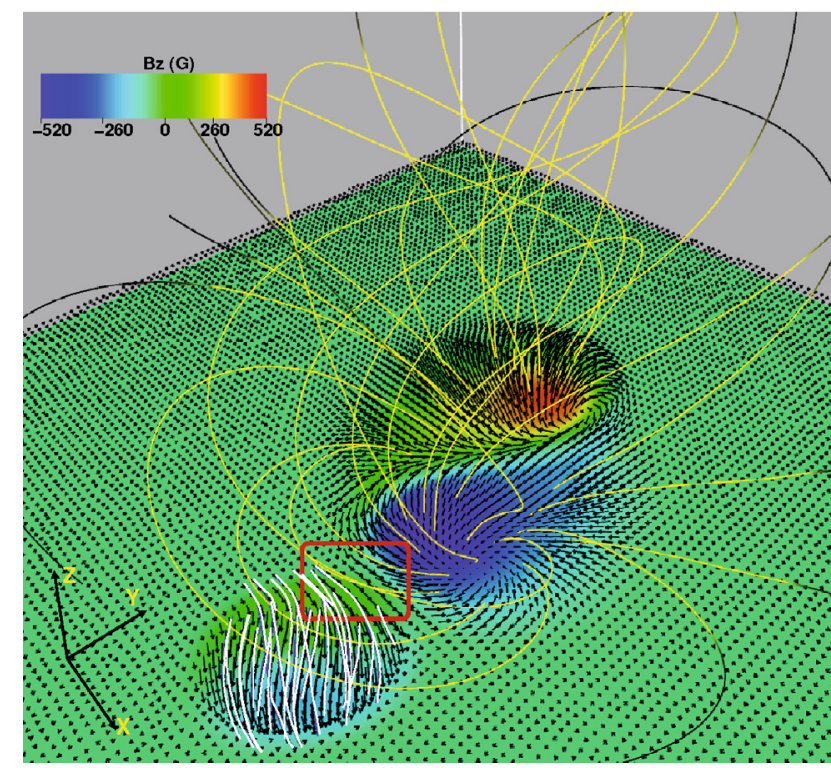

Fig. 2. Distribution of $B_{\mathrm{z}}$ (colormap), total magnetic field vector (arrows) and fieldlines at the photosphere, $t=15 \mathrm{~min}$. The red panel indicates the region presented in Fig. 3.

a field strength of $6.5 \mathrm{KG}$, a minor radius of $a=0.4 \mathrm{Mm}$ and a major radius $R \approx 4 \mathrm{Mm}$. The total longitudinal flux of the loop is $3.6 \times 10^{19} \mathrm{Mx}$, as in a small or ephemeral active region. At $t=0$ of our simulation, the upper part of the magnetic field of the loop is located 1.1 Mm below the photosphere. The second loop is located deeper within the convection zone. The horizontal $(x)$ distance between the two loops is $1.7 \mathrm{Mm}$. The second loop has a weaker field strength $(2.6 \mathrm{KG})$ and less flux $\left(1.8 \times 10^{18} \mathrm{Mx}\right)$. The crest of the field has to rise a distance of $2 \mathrm{Mm}$ to reach the photosphere. It has a minor radius of $a=0.25 \mathrm{Mm}$ and a major radius $R \approx 2 \mathrm{Mm}$.

When the first flux rope reaches the photosphere, at $t=$ $3.5 \mathrm{~min}$, a bipolar region is formed. Eventually, the two main polarities drift apart, moving along the y-direction. The magnetic field spreads out, forming a magnetic layer at the isothermal photosphere. Now, magnetic pressure builds up as more internal magnetic layers rise to the photosphere. This enhancement of magnetic pressure triggers the magnetic buoyancy instability and, thus, the field rises further into the non-magnetized corona. The lateral expansion creates an ambient magnetic field for the following emerging tube to rise into. The flux emergence of the second toroidal loop is followed by dynamical interaction (e.g., reconnection) between the upcoming and the ambient field.

Figure 2 shows the distribution of $B_{\mathrm{Z}}$ at the photosphere and the topology of the fieldlines around the active region. New magnetic flux (fieldlines in white) emerges close to one of the sides of the active region (yellow fieldlines): the positive polarity of the emerging field intersects the photosphere at the vicinity of the negative polarity of the preexisting field.

Eventually, the magnetic pressure force of the emerging field leads to the expansion of the field, which is pressed against the ambient field. A current layer is formed at the interface between the two magnetic flux systems. The value of the relative angle between the emerging fieldlines and the preexisting field changes over the dynamical evolution of the system in the three-dimensional space. It increases as the two polarities of the emerging field move away from each other along the $y$-direction.
A typical value of the relative angle at $t=20 \mathrm{~min}$ is $\approx 150 \mathrm{de}-$ grees and, thus, the orientation of the fieldlines becomes favorable for effective reconnection.

Figure 3 is a vertical cut at $x=1 \mathrm{Mm}$ and $t=20 \mathrm{~min}$, showing the distribution of $V_{\mathrm{z}}$ (left panel) and temperature (right panel). The full magnetic field vector and vertical velocity are overplotted (arrows) in the two panels. As reconnection occurs across the current structure, new links are established between the emerging and the pre-existing coronal field. Underneath the current layer, reconnected fieldlines adopt a loop-like shape and join the positive polarity of the emerging field with the negative polarity of the active region. At the upper edge, another set of reconnected fieldlines lie over the emerging field and couple the two remaining polarities.

Emission of a high velocity outflow occurs at the upper edge of the current layer. This is a reconnection jet, which is moving with speeds of around $100 \mathrm{~km} \mathrm{~s}^{-1}$ (which is comparable to the local Alfvén speed). The jet is magnetically driven and accelerated by the tension of the reconnected magnetic field lines. The flow, which is propagating downward from the reconnection site, reaches lower speeds $\left(\approx 30 \mathrm{~km} \mathrm{~s}^{-1}\right)$. There is a good correlation between the high velocity flows and hot plasma emission from the reconnection site. High temperature values of the order of $1 \mathrm{MK}$ are reached along the jet. The plasma density of the jet, shortly after the emission, is more than 10 times the density of the initial background plasma at the same height. Hot plasma is also found at the reconnection site and at the top of the flux pileup regime (with a loop-like shape) underneath the current layer. There, the plasma is also highly compressed and the temperature could reach values of a few MK during the evolution. The overall configuration resembles the "L-like" shape of the observed jet in Fig. 1.

The different magnetic domains in 3D are shown in Fig. 4. The emerging field lines (in blue) reconnect with the preexisting field (in yellow), producing a high-velocity, hot plasma emission. The isosurface (in red) represents temperature of $1 \mathrm{MK}$ along the jet, which extends over the interface between the interacting magnetic fields. High-temperature loops are formed when the hot plasma is ejected downward, at the crests of the low-lying reconnected fieldlines (in white). At larger heights, a new magnetic domain is formed (in green). It consists of new and long, reconnected fieldlines that join the two far ends of the emerging and the active region's field. At this stage of the evolution, the orientation of the jet is slightly oblique. This phase of emission, during which the jet keeps the same plasma properties, lasts for about $7 \mathrm{~min}$.

However, the shape and direction of the jet changes during the dynamical interaction of the two magnetic systems. The curvature of the surrounding, ambient fieldlines determines the direction of the jet. In the early stages of the evolution, the reconnected fieldlines above the jet (in green in Fig. 3, top panel) are oriented almost in a transverse direction to the jet. The tension of these fieldlines must be released for the jet to rise vertically and reach higher levels of the atmosphere. On the contrary, the numerical results show that the jet is trapped by the ambient field and it is compelled to move laterally, along the direction of the upper reconnected fieldlines that join the two systems. Now, the jet adopts an arch-like shape as it moves away (with speeds of around $100 \mathrm{~km} \mathrm{~s}^{-1}$ ) from the reconnection site and towards the preexisting magnetic domain. The bottom panel in Fig. 4 shows the connectivity of the magnetic lines around the reconnection site at $t=23 \mathrm{~min}$. High temperature $(\approx 2 \mathrm{MK})$ is shown by the red isosurface. 

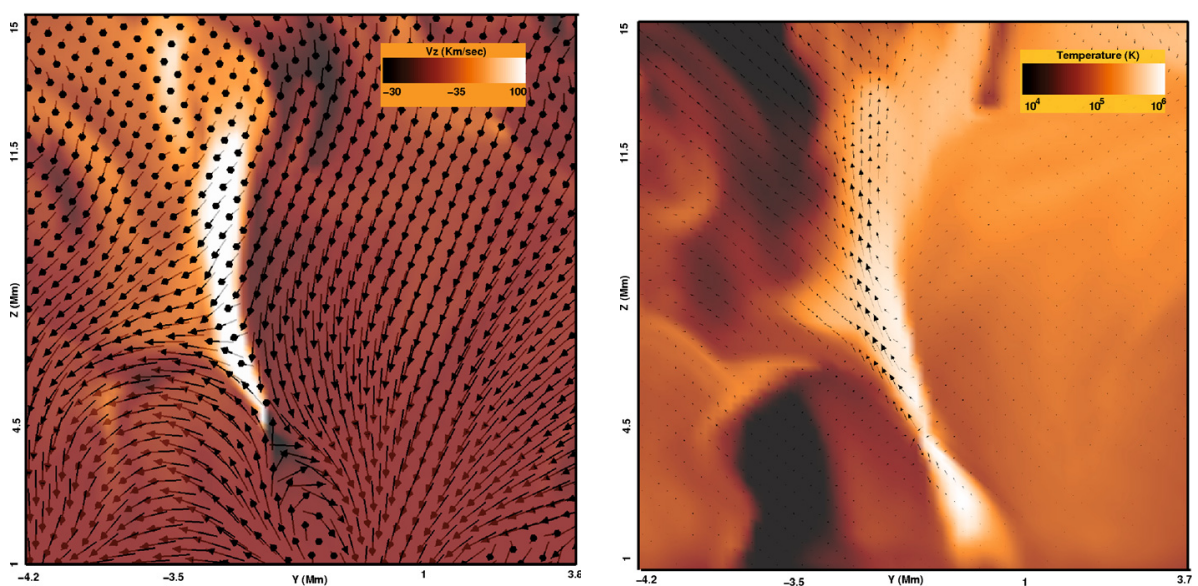

Fig. 3. Left: Distribution of the vertical velocity (colormap) and projection of the full magnetic field vector (arrows) onto the vertical $(x=1 \mathrm{Mm})$ plane. Right: distribution of temperature (colormap) and vertical velocity (arrows) onto the same plane. Time is, $t=20 \mathrm{~min}$.
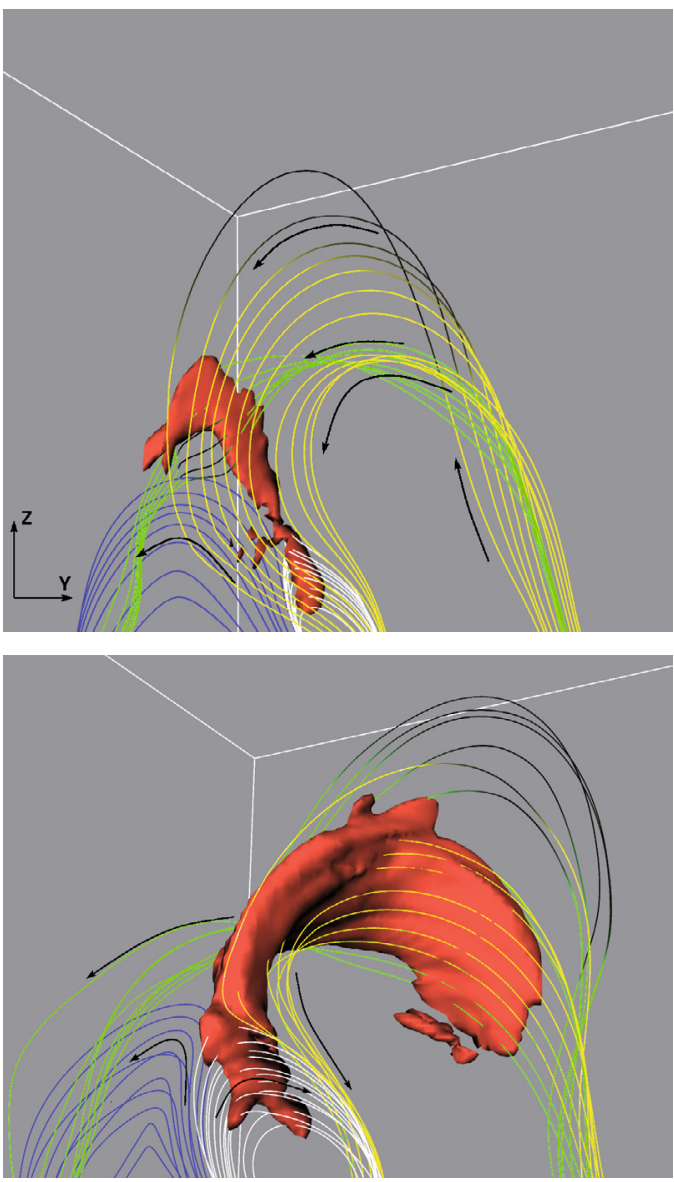

Fig. 4. 3D topology of fieldlines around the emission of the jet, at $t=20 \mathrm{~min}$ (top) and $t=23 \mathrm{~min}$ (bottom). Temperature is shown by the isosurface (in red). Arrows show the direction of the magnetic field lines.

\section{Discussion and conclusions}

The computed plasma presents a jet of $15 \mathrm{Mm}$ length and $1 \mathrm{Mm}$ width, presenting upflow velocities of $\simeq 100 \mathrm{~km} \mathrm{~s}^{-1}$ (Fig. 3 ). This agrees with observations where the jet has a length varying from 10 to $19 \mathrm{Mm}$, a width of $1.5 \mathrm{Mm}$ (Fig. $1 \mathrm{a}-\mathrm{f}$ ) and a velocity of $117 \mathrm{~km} \mathrm{~s}^{-1}$. The appearance of hot downflows along the reconnected loops of $\simeq 30 \mathrm{~km} \mathrm{~s}^{-1}$ is consistent with the redshifts of 10-20 $\mathrm{km} \mathrm{s}^{-1}$ measured on the bright structure in Ne VIII dopplergram (Fig. $1 \mathrm{~g}, \mathrm{~h}$ ). The simulation includes a cooler plasma component with smaller upflows of $\simeq 35 \mathrm{~km} \mathrm{~s}^{-1}$ on the right of the hot jet (Fig. 3) which corresponds to the jet observed in the C IV $1548 \AA$ line (Fig. 1 i, j) where the measured velocities are smaller. The simulations predict that, eventually, the jet is collimated along the reconnecting magnetic field lines that establish new connections (loops) between the emerging field and the field of the active region. TRACE 171 observations show sudden mass flows along loops, connecting the vicinity of the emerging flux region to the west side of the active region, triggered shortly after the first ejection of the hot plasma.

In our simulations, we did not include the effect of conduction. Thus, we cannot study whether evaporation flows, which might account for jets, form during the evolution of the system. The 3D geometric shape of the jets (see bottom panel in Fig. 4) reveals a bent structure. To show whether the jets adopt helical shapes undergoing untwisting while they are lifting off requires a detailed study of the fieldline topology during the temporal and spatial evolution of the jets. This important issue together with the recurrent appearance of the observed jets (a process that also occurs in our numerical experiments) will be addressed in a future study.

Acknowledgements. Financial support by the European Commission through the SOLAIRE network (MTRM-CT-2006-035484) is gratefully acknowledged.

\section{References}

Arnaud, M., \& Rothenflug, R. 1985, A\&AS, 60, 425

Arber, T. D., Longbottom, A. W., Gerrard, C. L., \& Milne, A. M. 2001, JCoPh., 171,151

Archontis, V., Moreno-Insertis, F., Galsgaard, K., \& Hood, A. W. 2005, ApJ, 635,1299

Canfield, R. C., Reardon, K. P., Leka, K. D., et al. 1996, ApJ, 464, 1016

Chae, J., Qiu, J., Wang, H., \& Goode, P. R. 1999, ApJ, 513, 75

Chifor, C., Young, P. R., Isobe, H., et al. 2008a, A\&A, 481, 57

Chifor, C., Isobe, H., Mason, H. E., et al. 2008b, A\&A, 491, 279

Gontikakis, C., Dara, H. C., Zachariadis, Th. G., et al. 2006, Sol. Phys., 233, 57

Hood, A. W., Archontis, V., Galsgaard, K., \& Moreno-Insertis, F. 2009, A\&A, 503,999

Moreno Insertis, F., Galsgaard, K., \& Uggarte-Urra, I. 2008, ApJ, 673, 211

Pariat, E., Antiochos, S., \& DeVore, C. R. 2009, ApJ, 691, 61

Patsourakos, S., Pariat, E., Vourlidas, A., et al. 2008, ApJ, 680, 73

Schmelz, J. T., Saba, J. L. R., Ghosh, D., \& Strong, K. T. 1996, ApJ, 473, 519

Shibata, K. 1996, ASP Conf. Ser., 111

Shimojo, M., Hashimoto, S., Shibata, K., et al. 1996, PASJ, 48, 123

Shimojo, M., Shibata, K., Yokoyama, T., et al. 2001, ApJ, 550, 1051

Teriaca, L., Banerjee, D., \& Doyle, J. G. 1999, A\&A, 349, 636

Tsinganos, K. 1980, ApJ, 239, 746

Yokoyama, T., \& Shibata, K. 1996, PASJ, 48, 353 